ELECTRONIC RESEARCH ANNOUNCEMENTS OF THE AMERICAN MATHEMATICAL SOCIETY

Volume 11, Pages 89-94 (November 15, 2005)

S $1079-6762(05) 00151-4$

\title{
INVARIANT MEASURES FOR THE HOROCYCLE FLOW ON PERIODIC HYPERBOLIC SURFACES
}

\author{
FRANÇOIS LEDRAPPIER AND OMRI SARIG
}

(Communicated by Boris Hasselblatt)

Pour Martine

\begin{abstract}
We describe the ergodic invariant Radon measures for the horocycle flow on general (infinite) regular covers of finite volume hyperbolic surfaces. The method is to establish a bijection between these measures and the positive minimal eigenfunctions of the Laplacian of the covering surface.
\end{abstract}

\section{INTRODUCTION}

Let $\mathbb{D}$ be the open unit disc equipped with the hyperbolic metric $2|d z| /\left(1-|z|^{2}\right)$, and let $T^{1}(\mathbb{D})$ denote the collection of unit tangent vectors to $\mathbb{D}$. A horocycle in $\mathbb{D}$ is a circle contained in $\overline{\mathbb{D}}$ which touches $\partial \mathbb{D}$ at one point. For every $v \in T^{1}(\mathbb{D})$ there is a unique horocycle which passes through the base point of $v$ and the terminus of geodesic determined by $v$. The horocycle flow of $\mathbb{D}$ is the flow $h^{t}: T^{1}(\mathbb{D}) \rightarrow T^{1}(\mathbb{D})$ which moves a unit tangent vector along the horocycle it determines in the positive direction, at unit speed.

A hyperbolic surface can be written in the form $M:=\Gamma \backslash \mathbb{D}$ where $\Gamma$ is a torsion free discrete subgroup of $M o ̈ b(\mathbb{D})$, the group of Möbius transformations of the disc. The horocycle flow and geodesic flow descend to flows $h, g$ on $T^{1}(M)$.

A famous theorem of Furstenberg $[\mathrm{F}$ says that if $M$ is compact, then $h$ has up to normalization a unique invariant Radon measure. Variants of this phenomena have been established for more general geometrically finite hyperbolic surfaces by Dani [D], Burger $[\mathrm{Bu}$, and Roblin [ Ro, and for more general actions by Ratner Ra. The geometrically infinite case is still almost completely open.

We contribute to the understanding of this case by treating the periodic surfaces

$$
M=\Gamma \backslash \mathbb{D} \text { where }\{i d\} \neq \Gamma \triangleleft \Gamma_{0}, \Gamma_{0} \text { is a torsion free lattice in } \operatorname{Möb}(\mathbb{D}) \text {. }
$$

$M$ is a regular cover of the finite volume surface $M_{0}=\Gamma_{0} \backslash \mathbb{D}$. We call $M_{0}$ the period of $M$. The group of deck transformations $G$ is called the symmetry group of $M$. A periodic surface is called cocompact if $M_{0}$ is compact.

The symmetry group is always finitely generated, because $G \simeq \Gamma_{0} / \Gamma$ and $\Gamma_{0}$ is finitely generated. Any finitely generated group can be realized as a symmetry

Received by the editors July 27, 2005.

2000 Mathematics Subject Classification. Primary 37D40, 37A40; Secondary 31C12.

F.L. is supported by NSF grant DMS-0400687.

O.S. is supported by NSF grant DMS-0500630.

(C)2005 American Mathematical Society Reverts to public domain 28 years from publication 
group of some surface. A periodic surface is called Abelian, nilpotent, etc. if its symmetry group is Abelian, nilpotent, etc.

It follows from the work of Ratner [Ra] that the horocycle flow has no finite invariant measures on periodic surfaces of infinite volume, other than measures supported on closed horocycles. But it has non-trivial invariant Radon measures, e.g. the volume measure on the unit tangent bundle 1

The ergodic invariant Radon measures (e.i.r.m.'s) for the horocycle flow on a periodic surface have so far only been classified for free Abelian cocompact surfaces. In this case every homomorphism $\varphi: G \rightarrow \mathbb{R}$ determines a unique (ray of) e.i.r.m. $m$ such that $m \circ d D=e^{\varphi(D)} m(D \in G)$ [BL, and every e.i.r.m. arises this way Sa. This is false for general periodic surfaces; see below.

We announce here the description of the horocycle e.i.r.m.'s for general periodic surfaces. For proofs and details see [LeS2].

\section{Preliminaries}

$K A N$-coordinates. Every $h$-e.i.r.m. on $T^{1}(M)$ lifts to some $\Gamma$-invariant $h$-invariant Radon measure on $T^{1}(\mathbb{D})$. This set can be identified with $(\partial \mathbb{D} \times \mathbb{R}) \times \mathbb{R}$ as follows: Let $o \in \mathbb{D}$ denote the origin. For every $e^{i \theta} \in \partial \mathbb{D}$ and $z \in \mathbb{D}$ let $\omega_{\theta}(z)$ be the line element based in $z$ which determines the geodesic which ends at $e^{i \theta}$. The identification is $\left(e^{i \theta}, s, t\right) \longmapsto\left(h^{t} \circ g^{s}\right)\left(\omega_{\theta}(o)\right)$.

It is well known that $g^{s} \circ h^{t}=h^{t e^{-s}} \circ g^{s}$. Therefore, in these coordinates

$$
\begin{aligned}
& h^{t}\left(e^{i \theta_{0}}, s_{0}, t_{0}\right)=\left(e^{i \theta_{0}}, s_{0}, t_{0}+t\right) ; \\
& g^{s}\left(e^{i \theta_{0}}, s_{0}, t_{0}\right)=\left(e^{i \theta_{0}}, s_{0}+s, t_{0} e^{-s}\right) .
\end{aligned}
$$

It follows that any $h$-invariant measure $m$ must be of the form $d \mu\left(e^{i \theta}, s\right) d t$.

Babillot's Program Ba. Suppose in addition that $m$ is quasi-invariant with respect to the geodesic flow.

In this case $m \circ g^{s}=e^{(\alpha-1) s} m$ for some $\alpha$ and all $s 2$ and we can decompose $m$ further into $d m=e^{\alpha s} d \nu\left(e^{i \theta}\right) d s d t$ for some finite measure $\nu$ on $\partial \mathbb{D}$. The measure $\nu$ can then be determined by the requirements that $m$ be $\Gamma$-invariant and $h$-ergodic. These requirements turn out to be equivalent to ergodicity and conformality with respect to the $\Gamma$-action on $\partial \mathbb{D}$ (see below).

This is the approach used by Martine Babillot in $\mathrm{Ba}$ to classify $h$-e.i.r.m. which are quasi-invariant with respect to the geodesic flow (for a different approach, see ASS).

\section{Results}

Some $h$-e.i.r.m.'s are not g-quasi-invariant: Take a non-cocompact periodic surface $M$ with period $M_{0}$. Since $M_{0}$ is a non-compact hyperbolic surface of finite volume, it has cusps. Every cusp is encircled by closed horocycles of finite length. These horocycles lift to $h$-orbits on $T^{1}(M)$. Lebesgue measure on them is $h$-ergodic, invariant, and Radon. But it is not $g$-quasi-invariant. We call these measures trivial $h$-e.i.r.m's. This is the only obstruction:

\footnotetext{
${ }^{1} \mathrm{~A}$ Radon measure is a measure which gives compact sets finite measure.

${ }^{2}$ If $m$ is $h$-e.i.r.m., then so is $m \circ g^{s}$ because $g^{s} \circ h^{t}=h^{t e^{-s}} \circ g^{s}$. Since $m, m \circ g^{s}$ are ergodic and equivalent, they must be proportional. The constant must of the form $e^{\beta s}$. Set $\beta=\alpha-1$.
} 
Theorem 1. Let $M$ be a periodic surface with period $M_{0}$. Any non-trivial h-e.i.r.m. on $T^{1}(M)$ is quasi-invariant with respect to the geodesic flow.

Let $\Gamma$ be a Fuchsian group, and $\nu$ some measure on $\partial \mathbb{D}$. We say that $\nu$ is $\Gamma$ ergodic if any $\Gamma$-invariant function is constant on a set of full measure. We say that $\nu$ is $\Gamma$-conformal (with parameter $\alpha$ ) if $\nu$ is finite, and $\frac{d \nu \circ g}{d \nu}=\left|g^{\prime}\right|^{\alpha}$ for all $g \in \Gamma$ (see $[\mathrm{Su}]$ ). Theorem 1 allows us to complete Babillot's program and show

Theorem 2. Let $M=\Gamma \backslash \mathbb{D}$ be a periodic surface. If $\nu$ is non-atomic, $\Gamma$-ergodic, and conformal with parameter $\alpha$, then $e^{\alpha s} d \nu\left(e^{i \theta}\right) d s d t$ is a $\Gamma$-invariant measure on $T^{1}(\mathbb{D})$, which projects to a non-trivial h-e.i.r.m on $T^{1}(\Gamma \backslash \mathbb{D})$. Any non-trivial $h$ e.i.r.m on $T^{1}(\Gamma \backslash \mathbb{D})$ is of this form.

Recall that the hyperbolic Laplacian of $\mathbb{D}$ is a second order differential operator on $C^{2}(\mathbb{D})$ such that $\Delta_{\mathbb{D}}(f \circ \varphi)=\left(\Delta_{\mathbb{D}} f\right) \circ \varphi$ for all $\varphi \in \operatorname{Möb}(\mathbb{D})$. This determines $\Delta_{\mathbb{D}}$ up to a constant. The invariance property of $\Delta_{\mathbb{D}}$ means that it descends to an operator $\Delta_{M}$ on $M=\Gamma \backslash \mathbb{D}$, called the hyperbolic Laplacian of $M$.

The collection of positive $\lambda$-eigenfunctions of $\Delta_{M}$ forms a cone. The extremal rays of this cone are the directions generated by minimal positive $\lambda$ - eigenfunctions: $\lambda$-eigenfunctions $F$ for which $\Delta_{M} G=\lambda G, 0 \leq G \leq F \Rightarrow \exists c$ such that $G=c F$.

If $P\left(e^{i \theta}, z\right):=\frac{1-|z|^{2}}{\left|e^{i \theta}-z\right|^{2}}$ (the Poisson kernel), then $P\left(e^{i \theta}, z\right)^{\alpha}$ is an $\alpha(\alpha-1)$ positive eigenfunction of $\Delta_{\mathbb{D}}$. Consequently, any $\Gamma$-invariant function of the form $\sum c_{k} P\left(e^{i \theta_{k}}, z\right)^{\alpha}, c_{k} \geq 0$ defines a positive eigenfunction of $\Delta_{M}$. We call these eigenfunctions trivial eigenfunctions. As it turns out, in this case $e^{i \theta_{k}}$ must all be fixed points of parabolic elements of $\Gamma$, so minimal trivial eigenfunctions may be thought of as generalized Eisenstein series.

Following Babillot $\mathrm{Ba}$, we consider the assignment

$$
m=e^{\alpha s} d \nu\left(e^{i \theta}\right) d s d t \longmapsto F_{m}(z):=\int_{\partial \mathbb{D}} P\left(e^{i \theta}, z\right)^{\alpha} d \nu\left(e^{i \theta}\right) .
$$

Theorem 3. Let $M$ be a periodic surface. The mapping (困) is a bijection between the non-trivial e.i.r.m's of $h$ on $T^{1}(M)$ and the non-trivial minimal positive eigenfunctions of $\Delta_{M}$. This bijection satisfies:

(1) $m \circ g^{s}=e^{(\alpha-1) s} m \Leftrightarrow \Delta_{M} F_{m}=\alpha(\alpha-1) F_{m}$;

(2) $m \circ d D=c m \Leftrightarrow F_{m} \circ D=c F_{m}$ for all $D$ in the symmetry group of $M$.

Remark. In the cocompact case there are no trivial $h$-e.i.r.m's and no non-trivial positive eigenfunctions.

\section{EXAMPLeS}

Example 1 (Furstenberg's Theorem $[\mathrm{F}]$ ). The horocycle flow of a compact hyperbolic surface is uniquely ergodic.

Proof. All positive eigenfunctions on a compact surface are constant.

Example 2 (Dani-Smillie Theorem [D, DS]). The ergodic invariant Radon measures for the horocycle flow on a hyperbolic surface of finite area are all finite, and consist of trivial measures and measures proportional to the volume measure.

Proof. The minimal positive eigenfunctions on a hyperbolic surface of finite volume are either trivial, or constant [JM]. 
Example 3 (Kaimanovich's Theorem Kai1]). The volume measure on a periodic surface is $h$-ergodic iff the surface is Liouville: all bounded harmonic functions are constant.

Proof. Kaimanovich proved this for all hyperbolic surfaces [Kai1]. In the periodic case: The volume measure on $T^{1}(\mathbb{D})$ is $d m=e^{s} d \lambda\left(e^{i \theta}\right) d s d t, \lambda=$ Haar measure on $\partial \mathbb{D}$. By Theorem 2, $\lambda$ is $\Gamma$-ergodic iff $m$ is ergodic, in which case (by theorem 3 ) $F_{m}(z)=\int_{\partial \mathbb{D}} P\left(e^{i \theta}, z\right) d \lambda\left(e^{i \theta}\right) \equiv 1$ is minimal. The minimality of 1 is the same as the Liouville property.

Example 4 (The strong Liouville property). The volume measure on a periodic surface is the unique $g$-invariant $h$-e.i.r.m. on $M$ iff the surface is strongly Liouville: all positive harmonic functions on the surface are constant.

Proof. $g$-invariant $h$-e.i.r.m's are necessarily non-trivial, and therefore correspond to minimal positive harmonic functions. The volume measure corresponds to the constant function.

Example 5 (Nilpotent surfaces). Let $M$ be a cocompact periodic surface with nilpotent symmetry group $G$. Every homomorphism $\varphi: G \rightarrow \mathbb{R}$ determines an $h$-e.i.r.m. measure $m$ (unique up to a constant) such that $m \circ d D=e^{\varphi(D)} m$ for every $D \in G$, and every $h$-e.i.r.m. is of this form.

Proof. This is because the minimal positive eigenfunctions for a cocompact nilpotent surface form a family $\left\{t F_{\varphi}: t>0, \varphi: G \rightarrow \mathbb{R}\right.$ is a homomorphism $\}$, where $F_{\varphi} \circ D=e^{\varphi(D)} F_{\varphi}$ for all $D \in G$ (see [LP], CG]). This example strengthens the main result of [Ba], ASS] by removing the $g$-quasi-invariance assumption.

Example 6 (Polynomial growth). Let $M$ be a cocompact periodic surface of polynomial growth 3 The symmetry group of $M$ contains a finitely generated normal nilpotent subgroup $N$ of finite index, and the rays of $h$-e.i.r.m.'s on $T^{1}(M)$ are in bijection with the homomorphisms from $N$ to $\mathbb{R}$.

Proof. The symmetry group of a surface of polynomial growth is a group of polynomial growth. By Gromov's theorem Gr. it contains a normal nilpotent subgroup $N$ of finite index. It is now possible to represent $M$ as a nilpotent cover of some compact hyperbolic surface $M_{1}$ (we thank Y. Coudene for this observation). This reduces Example 5 to Example 4.

Remark. This shows that the $h$-e.i.r.m.'s on a cocompact periodic surface of polynomial growth can be naturally parameterized as a $d$-parameter family with $d=$ $\operatorname{rank}(N /[N, N])$ (where the rank of the finitely generated Abelian group $A=$ $N /[N, N]$ is the $d$ in $\left.A / \operatorname{Tor}(\mathrm{A}) \simeq \mathbb{Z}^{d}\right)$.

Example 7 (Polycyclic surfaces). Cocompact polycyclic surfaces which are not virtually nilpotent are Liouville, but not strongly Liouville 4 Therefore,

\footnotetext{
${ }^{3}$ A Riemannian surface is said to be of polynomial growth if the volume of balls of radius $R$ is $O\left(R^{\delta}\right)$ for some $\delta$ as $R \rightarrow \infty$.

${ }^{4} G$ is polycyclic if $\exists G_{i} \triangleleft G$ such that $\{1\}=G_{0} \triangleleft \cdots \triangleleft G_{n}=G$ and $G_{i} / G_{i-1}$ are cyclic. Polycyclic groups are characterized as the solvable groups all of whose subgroups are finitely generated. $G$ is virtually nilpotent if $\exists N \triangleleft G$ nilpotent such that $|G / N|<\infty$. Finitely generated virtually nilpotent groups are characterized as the groups of polynomial growth: if $\Lambda$ is a finite set of generators, then $\left|\Lambda^{n}\right|=O\left(n^{\alpha}\right)$ for some $\alpha$.
} 
(i) The volume measure on $T^{1}(M)$ is a $g$-invariant $h$-e.i.r.m.;

(ii) There are other $g$-invariant $h$-e.i.r.m.'s., and these measures are not quasiinvariant with respect to all deck transformations.

Proof. A cocompact polycyclic surface has the Liouville property (Kaimanovich [Kai2]), and we saw that this implies (i). Polycyclic groups are linear, therefore the work of Bougerol and Élie BE] provides a non-constant minimal positive harmonic function $F$ on $M$. The measure corresponding to $F$ is $g$-invariant, because $F_{0}$ has eigenvalue zero. It cannot be quasi-invariant with respect to all deck transformations, otherwise it would be invariant with respect to the commutator of the symmetry group, and would therefore define a $g$-invariant $h$-e.i.r.m on the homology cover of the period of $M$, different from the volume measure. But the homology cover of a compact surface is Liouville [LS], and therefore does not admit such measures.

Note the gradual deterioration in measure rigidity as the symmetry group 'grows'.

Final comments. We see that the horocycle flow on an infinite periodic surface usually has more than one invariant Radon measure. It is natural to ask for an ergodic theoretic property which singles out one e.i.r.m out of the multitude ('conditional unique ergodicity'). There are indications that this can be done for periodic surfaces of polynomial growth. See [LeS1] for a treatment of $\mathbb{Z}^{d}$-covers.

\section{REFERENCES}

[ASS] J. Aaronson, O. Sarig, R. Solomyak: Tail-invariant measures for some suspension semiflows. Discr. and Contin. Dyn. Sys. 8 (no. 3), 725-735 (2002). MR1897877|(2003f:37009)

[Ba] M. Babillot: On the classification of invariant measures for horospherical foliations on nilpotent covers of negatively curved manifolds. In: Random walks and geometry (V.A. Kaimanovich, Ed.), de Gruyter, Berlin 2004, 319-335. MR2087786 (2005k:37058)

[BL] M. Babillot and F. Ledrappier: Geodesic paths and horocycle flows on Abelian covers. Lie groups and ergodic theory (Mumbai, 1996), 1-32, Tata Inst. Fund. Res. Stud. Math. 14, Tata Inst. Fund. Res., Bombay, 1998. MR.1699356 (2000e:37029)

[BE] P. Bougerol and L. Élie: Existence of positive harmonic functions on groups and on covering manifolds. Ann. Inst. H. Poincaré Probab. Statist. 31 (1995), no. 1, 59-80. MR:1340031 (96g:60011)

[Bu] M. Burger: Horocycle flow on geometrically finite surfaces. Duke Math. J. 61 (1990), no. 3, 779-803. MR.1084459 (91k:58102)

[CG] J.-P. Conze and Y. Guivarc'h: Propriété de droite fixe et fonctions propres des opérateurs de convolution. Séminaire de Probabilités, I (Univ. Rennes, Rennes, 1976), Exp. No. 4, 22 pp. Dept. Math. Informat., Univ. Rennes, Rennes, 1976. MR0487265 (58:6916)

[D] S. G. Dani: Invariant measures of horospherical flows on non-compact homogeneous spaces. Invent. Math. 47 (1978), no. 2, 101-138. MR0578655 (58:28260)

[DS] S. G. Dani and J. Smillie: Uniform distribution of horocycle orbits for Fuchsian groups. Duke Math. J. 51 (1984), 185-194. MR0744294 (85f:58093)

[F] H. Furstenberg: The unique ergodicity of the horocycle flow. Springer Lecture Notes 318 (1972), 95-115. MR0393339 (52:14149)

[Gr] M. Gromov: Groups of polynomial growth and expanding maps. Inst. Hautes Études Sci. Publ. Math. No. 53 (1981), 53-73. MR0623534 (83b:53041)

[JM] L. Ji and R. MacPherson: Geometry of compactifications of locally symmetric spaces. Ann. Inst. Fourier (Grenoble) 52 (2002), no. 2, 457-559. MR1906482 (2004h:22006)

[Kai1] V. Kaimanovich: Ergodic properties of the horocycle flow and classification of Fuchsian groups. J. Dynam. Control Systems 6 (2000), no. 1, 21-56. MR.1738739 (2000m:37033)

[Kai2] V. Kaimanovich: Brownian motion and harmonic functions on covering manifolds. An entropic approach. Dokl. Akad. Nauk SSSR 288 (1986), no. 5. Engl. Transl. in Soviet Math. Doklady 33 (1986), 812-816. MR0852647 (88k:58163) 
[LP] V. Lin and Y. Pinchover: Manifolds with group actions and elliptic operators. Memoirs of the AMS 112 (1994), 78pp. MR1230774 (95d:58119)

[LS] T. Lyons and D. Sullivan: Function theory, random paths and covering spaces. J. Differential Geom. 19 (1984), no. 2, 299-323. MR0755228 (86b:58130)

[LeS1] F. Ledrappier and O. Sarig: Unique ergodicity for non-uniquely ergodic horocycle flows. To appear in Disc. Cont. Dynam. Syst. Issue dedicated to A. Katok.

[LeS2] F. Ledrappier and O. Sarig: Invariant measures for the horocycle flow on periodic hyperbolic surfaces. Preprint available at http://www.math.psu.edu/sarig/

[Ra] M. Ratner: On Raghunathan's measure conjecture. Ann. of Math. (2) 134 (1991), no. 3, 545-607. MR 1135878 (93a:22009)

[Ro] T. Roblin: Un théorème de Fatou pour les densités conformes avec applications aux revêtements galoisiens en courbure négative. Preprint.

[Sa] O. Sarig: Invariant measures for the horocycle flow on Abelian covers. Inv. Math. 157 (2004), 519-551. MR2092768 (2005k:37059)

[Su] D. Sullivan: The density at infinity of a discrete group of hyperbolic motions. Inst. Hautes Études Sci. Publ. Math. 50 (1979), 171-202. MR0556586 (81b:58031)

Department of Mathematics, University of Notre-Dame, Notre-Dame, IN 46556-4618

E-mail address: ledrappier.1@nd.edu

Mathematics Department, Pennsylvania State University, University Park, PA 16802

E-mail address: sarig@math.psu.edu 